\title{
The Higgs program and open questions in particle physics and cosmology
}

\author{
Beate Heinemann ${ }^{1,2 a}$, and Yosef $\mathrm{Nir}^{3 b 1}$ \\ ${ }^{11}$ Deutsches Elektronen-Synchrotron, 22607 Hamburg, Germany \\ ${ }^{2}$ Albert-Ludwigs-Universität Freiburg, Physikalisches Institut, 79104 Freiburg, Germany \\ ${ }^{3}$ Department of Particle Physics and Astrophysics, \\ Weizmann Institute of Science, Rehovot, Israel 7610001*
}

\begin{abstract}
The Higgs program is relevant to many of the open fundamental questions in particle physics and in cosmology. Thus, when discussing future collider experiments, one way of comparing them is by assessing their potential contributions to progress on these questions. We discuss in detail the capabilities of the various proposed experiments in searching for singlet scalars, which are relevant to several of the open questions, and in measuring Higgs decays to fermion pairs, which are relevant to the flavor puzzles. On other interesting questions, we list the most relevant observables within the Higgs program.
\end{abstract}

\section{INTRODUCTION}

The jewel in the crown of the achievements of the LHC experiments to date is the Higgs boson discovery in July $2012[1,2]$. The discovery of a Higgs boson is, however, not just an end of a story - a quest that began with the theoretical predictions of Brout, Englert [3] and Higgs [4] - but also a beginning of one. While the presence of the Higgs boson provides a solution to the question of how fundamental particles can acquire mass, it does not actually explain the mass values themselves, and it also raises new questions. "The Higgs program" is a major research project at the LHC, and at all proposed future collider experiments. From the experimental side, the Higgs program refers to a large set of measurements aimed to learn the detailed properties of this unique particle. From the theoretical side, this is also a very exciting program, as it touches upon several open questions and puzzles in particle physics and particle cosmology.

In this article, we explore the relationship of the properties of the Higgs boson to big questions in the field of particle physics and cosmology. This work was initiated by discussions in the Scientific Policy Committee of CERN in the context of discussions on future accelerators. All proposed future accelerators consider measurements of the Higgs boson a major part of their scientific program, and this short article is designed to summarize concisely the conclusions from the currently available literature that relates Higgs precision measurements to fundamental open questions about our Universe. We also summarize the precision these future colliders estimate, and try to contrast that with the precision required to answer a given question. It is worth noting that for many of the questions there are also important observables unrelated to the Higgs boson measurements (at both collider and non-collider experiments), but discussing those is beyond the scope of this article.

Here is a list of seven open questions at the forefront of particle physics and cosmology, to which the Higgs program may provide answers:

1. Is $h$ the only scalar degree of freedom?

2. Is $h$ elementary?

3. What keeps $m_{h}^{2} \ll m_{\mathrm{Pl}}^{2}$ ?

4. Was the electroweak phase transition first order?

5. Did CP violating $h$ interactions generate the baryon asymmetry?

6. Are there light SM-singlet degrees of freedom (in particular, related to Dark Matter)?

7. What is the solution of the flavor puzzle(s)?

In what follows, we focus on two topics. In Section III, we describe the search for singlet scalars. We choose this topic for four reasons:

\footnotetext{
*Electronic address: ${ }^{a}$ beate.heinemann@desy.de; ${ }^{b}$ yosef.nir@weizmann.ac.il
} 
- It is relevant to a large number (four) of the above questions;

- The Higgs program is likely to be the unique portal to such particles;

- The search for singlet scalars by itself constitutes a rich and broad experimental program;

- For the question of the electroweak phase transition, there is a clear target for the required accuracy that will give a definite answer on this question.

In Section IV, we discuss the measurements that are relevant to the flavor puzzles. Again, there are several reasons for this choice of topic:

- The flavor puzzles are long standing, and the Higgs program provides an opportunity to measure new flavor parameters and make progress;

- The main ingredient in the flavor-related Higgs program - the measurement of Yukawa couplings - is also relevant to other questions.

- The flavor related measurements by themselves constitute a rich and broad experimental program;

- In contrast to the question of electroweak phase transition, for the flavor measurements there is no lower bound on the size of new physics effects. Instead, the better accuracy, the higher the scale of new physics to which there is sensitivity.

All other topics are discussed briefly in Section V, where we describe the most relevant experimental measurements to each physics question, but do not compare the capabilities of the various experiments (which is still work in progress). In Section VI we present three important conclusions about the scientific significance of the Higgs program.

\section{EXPERIMENTS}

For the purpose of discussing the expected accuracy of future measurements, the following future collider parameters (integrated luminosity $\mathcal{L}$, center of mass energy $\sqrt{s}$, and, where relevant, polarization) are assumed:

- HL-LHC: $p p$ collider, $\mathcal{L}=3-4 \mathrm{ab}^{-1}$ at $\sqrt{s}=14 \mathrm{TeV}$ (2026 to late 2030s: $\gtrsim 10$ years) [5].

- ILC: $e^{+} e^{-}$collider, with $e^{-} 80 \%$ polarized and $e^{+} 30 \%$ polarized, to be operated in two stages (22 years total including shutdowns) [6]:

1. ILC250: $\mathcal{L}=2 \mathrm{ab}^{-1}$ at $\sqrt{s}=250 \mathrm{GeV}$ (11 years),

2. ILC500: $\mathcal{L}=4 \mathrm{ab}^{-1}$ at $\sqrt{s}=500 \mathrm{GeV}$ (10 years). This includes also a 1 -year run at $\sqrt{s}=365 \mathrm{GeV}$.

- CLIC: $e^{+} e^{-}$collider, with $e^{-} 80 \%$ polarized and $e^{+}$not polarized, to be operated in three stages (26 years including shutdowns) [5]:

1. CLIC380: $\mathcal{L}=1.0 \mathrm{ab}^{-1}$ at $\sqrt{s}=380 \mathrm{GeV}$ (8 years),

2. CLIC1500: $\mathcal{L}=2.5 \mathrm{ab}^{-1}$ at $\sqrt{s}=1400 \mathrm{GeV}$ (7 years),

3. CLIC3000: $\mathcal{L}=5.0 \mathrm{ab}^{-1}$ at $\sqrt{s}=3000 \mathrm{GeV}$ (7 years).

- CEPC: $e^{+} e^{-}$collider, without polarization, $\mathcal{L}=5.6 \mathrm{ab}^{-1}$ at $\sqrt{s}=240 \mathrm{GeV}$ (7 years) [7]. It is assumed that there are two experiments collecting the data, and the datasets are combined.

- FCC-ee: $e^{+} e^{-}$collider, without polarization, to be operated in two stages [5]:

1. FCC240: $\mathcal{L}=5.0 \mathrm{ab}^{-1}$ at $\sqrt{s}=240 \mathrm{GeV}$ (3 years).

2. FCC365: $\mathcal{L}=1.5 \mathrm{ab}^{-1}$ at $\sqrt{s} \approx 365 \mathrm{GeV}(5$ years $)$.

In both cases it is assumed that there are two experiments collecting the data, and the datasets are combined.

- LHeC: ep collider, colliding the $7 \mathrm{TeV}$ HL-LHC protons with $60 \mathrm{GeV}$ electrons, aiming to deliver $\mathcal{L}=1$ ab ${ }^{-1}$ within 12 years. For the last 4 years the HL-LHC is assumed not to operate concurrently for $p p$ collisions [5].

- HE-LHC: $p p$ collider, $\mathcal{L}=10 \mathrm{ab}^{-1}$ at $\sqrt{s}=27 \mathrm{TeV}$ (20 years) [5]. For the numbers presented in Table VII, $\mathcal{L}=15 \mathrm{ab}^{-1}$ is considered, corresponding to two collider experiments combining at least $75 \%$ of the data [8]. 
- FCC-hh: $p p$ collider, $\mathcal{L}=20 \mathrm{ab}^{-1}$ at $\sqrt{s}=100 \mathrm{TeV}$ (25 years) [5]. For the numbers presented in Table VII, $\mathcal{L}=30 \mathrm{ab}^{-1}$ is considered, corresponding to two collider experiments combining at least $75 \%$ of the data [8].

- Muon collider: a muon collider is also a very interesting option to collide leptons at very high energies. This is, however, not considered in this report as the studies related to this are currently less advanced.

\section{SINGLET SCALARS}

One of the experimentally most challenging extensions of the Standard Model (SM), yet one that is relevant to many interesting Higgs-related questions, is that of a singlet scalar. In fact, it appears with relation to four of the seven open questions that we posed:

1. Is $h$ alone?

2. What keeps $m_{h}^{2} \ll m_{\mathrm{Pl}}^{2}$ ?

3. Was the electroweak phase transition first order?

4. Are there light SM-singlet degrees of freedom?

The simplest extension is to add to the SM a real scalar field $S(x)$, in the $(1,1)_{0}$ (gauge-singlet) representation. The most general renormalizable Lagrangian is [9-11] (we use the notations of [9])

$$
\mathcal{L}=\mathcal{L}_{\mathrm{SM}}+\frac{1}{2}\left(\partial_{\mu} S\right)\left(\partial^{\mu} S\right)-\frac{m_{s}^{2}}{2} S^{2}-\frac{a_{s}}{3} S^{3}-\frac{\lambda_{s}}{4} S^{4}-\lambda_{h s} \Phi^{\dagger} \Phi S^{2}-2 a_{h s} \Phi^{\dagger} \Phi S,
$$

where $\Phi$ is the Higgs doublet in the $(1,2)_{+1 / 2}$ representation. In the general case, both $\Phi$ and $S$ can acquire VEVs:

$$
\langle\Phi\rangle=(0, v / \sqrt{2}), \quad\langle S\rangle=v_{s},
$$

and the Higgs and singlet fields mix:

$$
\sin 2 \theta=\frac{4 v\left(a_{h s}+\lambda_{h s} v_{s}\right)}{M_{h}^{2}-M_{s}^{2}}
$$

where $M_{h}$ and $M_{s}$ are the mass eigenvalues of the two scalar mass eigenstates $\left(M_{h} \simeq 125 \mathrm{GeV}\right)$.

Deviations from the SM predictions for various couplings are often parameterized by " $\kappa$ parameters":

$$
\kappa_{x} \equiv \frac{g_{h x x}}{g_{h x x}^{S M}} .
$$

Within the SM extended by singlet scalars, the couplings of the Higgs boson to all fermion and vector boson pairs are modified by a universal factor. (For a discussion, see Section I of Ref. [10].) Defining $\delta g_{h}$ via

$$
\kappa_{x} \equiv 1+\delta g_{h},
$$

we have [9]

$$
\delta g_{h} \approx(\cos \theta-1)-\frac{\left|a_{h s}+\lambda_{h s} v_{s}\right|^{2}}{8 \pi^{2}} I_{B}\left(M_{h}^{2} ; M_{h}^{2}, M_{s}^{2}\right)-\frac{\left|\lambda_{h s}\right|^{2} v^{2}}{16 \pi^{2}} I_{B}\left(M_{h}^{2} ; M_{s}^{2}, M_{s}^{2}\right),
$$

where [12]

$$
I_{B}\left(p^{2} ; m_{1}^{2}, m_{2}^{2}\right)=\int_{0}^{1} d x \frac{x(1-x)}{x(1-x) p^{2}-x m_{1}^{2}-(1-x) m_{2}^{2}} .
$$

As concerns Eq. (6), we emphasize the following points:

- The first term on the right hand side (RHS) is a consequence of the mixing (see Eq. 3).

- The second and third terms are a consequence of the wave function renormalization of the Higgs at one loop. 
Usually, the first term dominates. This is not the case, however, in models where a $Z_{2}$ symmetry, under which $S$ $(\Phi)$ is odd (even), is imposed. This scenario is considered the most difficult one for finding experimental evidence for first order electroweak phase transition [11]. In this model, we have $a_{h s}=v_{s}=0$ and consequently $\theta=0$, so that the first two terms of Eq. (6) vanish. It follows that [12]

$$
\delta g_{h}^{\left(Z_{2}\right)}=-\frac{\left|\lambda_{h s}\right|^{2} v^{2}}{16 \pi^{2}} I_{B}\left(M_{h}^{2} ; M_{s}^{2}, M_{s}^{2}\right)=-\frac{\left|\lambda_{h s}\right|^{2} v^{2}}{16 \pi^{2} M_{h}^{2}}\left[1-\frac{4 M_{s}^{2} \arctan \left(\sqrt{\frac{M_{h}^{2}}{4 M_{s}^{2}-M_{h}^{2}}}\right)}{\sqrt{M_{h}^{2}\left(4 M_{s}^{2}-M_{h}^{2}\right)}}\right] .
$$

In the limit that $M_{s}^{2} \gg M_{h}^{2}[12]$,

$$
\delta g_{h}^{\left(Z_{2}\right)} \rightarrow+\frac{\left|\lambda_{h s}\right|^{2} v^{2}}{96 \pi^{2} M_{s}^{2}}
$$

A. $M_{s}<M_{h} / 2$

If $M_{s}<M_{h} / 2$, then a new decay channel for the Higgs boson opens up, $h \rightarrow S S$, with

$$
\Gamma(h \rightarrow S S)=\frac{\lambda_{h S S}^{2}}{8 \pi M_{h}} \sqrt{1-\frac{4 M_{s}^{2}}{M_{h}^{2}}},
$$

and

$$
\lambda_{h S S}=+c_{\theta}^{3} \lambda_{h s} v+c_{\theta}^{2} s_{\theta}\left(a_{s}+3 \lambda_{s} v_{s}+2 \lambda_{h s} v_{s}-2 a_{h s}\right)+c_{\theta} s_{\theta}^{2}\left(3 \lambda_{h} v-2 \lambda_{h s} v\right)+s_{\theta}^{3}\left(\lambda_{h s} v_{s}+a_{h s}\right) .
$$

In the $Z_{2}$ limit

$$
\lambda_{h S S}=\lambda_{h s} v
$$

and, furthermore, $S$ is stable, and therefore, the $h \rightarrow S S$ mode contributes to $h \rightarrow$ invisible. Ref. [13] collected the upper bounds and projections on $\mathrm{BR}(h \rightarrow$ invisible $)$. We quote updated results and future projections in Table I.

The $\operatorname{BR}(h \rightarrow$ invisible $)$ is determined directly by analyses searching for a Higgs boson produced in association with a $Z$ boson (at lepton and hadron colliders) or with forward jets (in VBF process at hadron colliders), and the Higgs boson decaying invisibly which is observed as missing transverse momentum in the experiments. Several $e^{+} e^{-}$colliders are able to probe this with a precision of $0.3 \%$. The best sensitivity is achieved by FCC $h h$ with $0.025 \%$.

In the case that no $Z_{2}$ symmetry is imposed and, in particular, $\sin \theta \neq 0$, the mass eigenstate $S$ inherits the Higgs couplings, suppressed by $\sin \theta$ :

$$
\lambda_{S f \bar{f}}=\sin \theta y_{f}^{\mathrm{SM}}
$$

where $y_{f}^{\mathrm{SM}}$ is the corresponding SM Yukawa coupling. The $h \rightarrow S S$ decay will be followed by $S \rightarrow f \bar{f}$ where $m_{f}<M_{s} / 2$ with coupling given by Eq. (13). There are two complementary ways of searching for these decays. One is the direct search for the final four fermion state, which we discuss further below. The second is the indirect search, via a global fit to the Higgs couplings, which can reveal (or constrain) a contribution to the Higgs width that is not accounted for by the final states that are included in the fit $[13,18,19]$, the so-called

$$
h \rightarrow \text { undetected. }
$$

Upper bounds on $\mathrm{BR}(h \rightarrow$ undetected $)$ are given in Table I, and put an upper bound on $\sin ^{2} \theta$ for a given $M_{s}$. The branching ratios to "undetected" final state are derived from the observed cross sections and branching ratios using certain constraints. For the LHC and HL-LHC this can only be done if $\kappa_{Z} \leq 1.0$ is assumed, which holds for singlets with $M_{s}<M_{h} / 2$ if the dominant effect is due to $\sin \theta \neq 0$ (and in general only holds for a fraction of new physics models). For the $e^{+} e^{-}$colliders it is possible to use a much more direct and very model-independent constraint [13] $\mathrm{BR}(h \rightarrow$ undetected $) \leq 1-\left(1-2 \delta_{\kappa}\right)^{2}$, where $\delta_{\kappa}$ is the precision on the measurement of $\kappa_{Z}$ (CLIC380: 0.4\%, CEPC: $0.25 \%$, ILC: $0.38 \%$, FCCee: $0.22 \%$ ).

Examples of other relevant experimental searches are collected in Table II. For the $\mu \mu b b$ final state it is expected that the HL-LHC [20] will have a sensitivity down to $\mathrm{BR}\left(h \rightarrow \mu^{+} \mu^{-} b \bar{b}\right) \sim 5 \times 10^{-5}$. For the other decays no projections are available. 
Table I: Current upper bounds and projections on $\mathrm{BR}_{\mathrm{inv}} \equiv \mathrm{BR}(h \rightarrow$ invisible $)$ and $\mathrm{BR}_{\text {und }} \equiv \mathrm{BR}(h \rightarrow$ undetected $)$. All upper bounds are given at 95\% CL unless explicitly stated. The LHC2 line updates the most recent bounds from CMS [14] and ATLAS [15]. Projections for future colliders are also shown: CLIC [16], CEPC [7], ILC [17], FCC ee $_{\text {and FCC }}$ hh [8]. For FCC $_{\mathrm{hh}}$ the SM background from $H \rightarrow 4 \nu$, corresponding to a BR of $0.113 \%$, has been subtracted and the limit refers to additional invisible new physics contributions. For the LHC and HL-LHC BR und is inferred assuming $\kappa_{Z} \leq 1$. The symbol " -" means that no value is available in the literature.

\begin{tabular}{|c|c|c||c|c|}
\hline \hline Collider & $\sqrt{s}[\mathrm{TeV}]$ & $\mathcal{L}\left[\mathrm{ab}^{-1}\right]$ & $\mathrm{BR}_{\text {inv }}[\%]$ & $\mathrm{BR}_{\text {und }}[\%]$ \\
\hline \hline LHC1 & 7,8 & 0.022 & 37 & 20 \\
LHC2 & 13 & 0.036 & 26 & - \\
LHC3 & 13 & 0.300 & $8.8(68 \%)$ & $7.6(68 \%)$ \\
HL-LHC & 14 & 3 & 2.6 & 2.5 \\
\hline \hline CLIC & 0.38 & 1 & $0.69(90 \%)$ & 1.6 \\
CEPC & 0.25 & 5.6 & 0.3 & 1.0 \\
ILC & 0.25 & 2 & 0.3 & 1.5 \\
FCC $_{\text {ee }}$ & 0.24 & 5 & 0.3 & 0.87 \\
FCC $_{\mathrm{hh}}$ & 100 & 20 & 0.025 & - \\
\hline \hline
\end{tabular}

Table II: Upper bounds on $\left(\sigma_{h} / \sigma_{h}^{\mathrm{SM}}\right) \times \mathrm{BR}\left(h \rightarrow S S \rightarrow \overline{f_{1}} f_{1} \overline{f_{2}} f_{2}\right)$.

\begin{tabular}{|c|c|c|c|}
\hline \hline$\overline{f_{1}} f_{1} \overline{f_{2}} f_{2}$ & $M_{s}[\mathrm{GeV}]$ & Upper bound & Ref. \\
\hline \hline$\tau \tau b b$ & $15-60$ & $0.03-0.12$ & CMS [21] \\
$\mu \mu b b$ & $25-63$ & $(1-8) \times 10^{-4}$ & CMS [22], ATLAS [23] \\
$\tau \tau \tau \tau$ & $9-15$ & $0.2-0.3$ & CMS [22] \\
$\mu \mu \tau \tau$ & $25-63$ & $(1-5) \times 10^{-4}$ & CMS [22, 24] \\
$\gamma \gamma j j$ & $20-60$ & $0.034-0.173$ & ATLAS [25] \\
\hline \hline
\end{tabular}

The total width of $h$ is, on one hand, suppressed by the suppression of the $h$-couplings to pairs of SM particles but, on the other hand, enhanced by the addition of the $h \rightarrow S S$ channel. It thus provides an interesting observable to probe this scenario.

The width of the $h$ boson can be inferred at the LHC using three methods:

- The cross section of off-shell $h$-boson production is proportional to $\left[\left(s-m_{h}^{2}\right)^{2}+m_{h}^{2} \Gamma_{H}^{2}\right]^{-1}$. Measuring it at different values of $s$ allows an extraction of the width under the assumption that the couplings of $h$ to the particles involved are independent of $s$ (this is not a good assumption for several BSM models). The most precise extraction [26] has been done in the $Z Z$ decay mode by the CMS collaboration using $\sim 80 \mathrm{fb}^{-1}$ of run-2 data, and gives $3.2_{-2.2}^{+2.8} \mathrm{MeV}$. It is foreseen that, with the HL-LHC and improvements in the theoretical calculations, $\Gamma_{H}$ will be extracted with a precision of $20 \%$ using this method [27].

- Using the diphoton decay mode it is also possible to determine the width through interference effects. This is complementary, as well as theoretically robust, and will be able to probe values of $(8-22) \times \Gamma_{h}[27$.

- Using the coupling measurements and assuming that $\kappa_{Z} \leq 1$ (which holds if the dominant effect is due to $\sin \theta \neq 0), \Gamma_{h}$ can be extracted with a precision of $5 \%$ with the HL-LHC. This determination of the width is only possible if one assumes that there are no undetected final states. We can either fix the width and determine the BR to undetected particles or we can set the latter to zero and determine the width.

In $e^{+} e^{-}$colliders, $\Gamma_{h}$ can be measured based on $\sigma\left(e^{+} e^{-} \rightarrow Z h\right)$ and $\operatorname{BR}\left(h \rightarrow Z Z^{*}\right)$ :

$$
\frac{\sigma\left(e^{+} e^{-} \rightarrow Z h\right)}{\mathrm{BR}\left(h \rightarrow Z Z^{*}\right)}=\frac{\sigma\left(e^{+} e^{-} \rightarrow Z h\right)}{\Gamma\left(h \rightarrow Z Z^{*}\right) / \Gamma_{h}}=\left[\frac{\sigma\left(e^{+} e^{-} \rightarrow Z h\right)}{\Gamma\left(h \rightarrow Z Z^{*}\right)}\right]_{\mathrm{SM}} \times \Gamma_{h},
$$




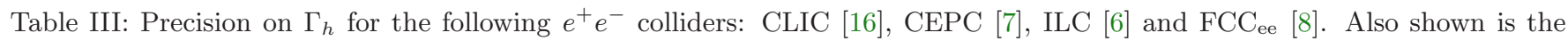
method used to determine the width.

\begin{tabular}{|c|c|c||c|c|}
\hline \hline Collider & $\sqrt{s}[\mathrm{TeV}]$ & $\mathcal{L}\left[\mathrm{ab}^{-1}\right]$ & $\delta \Gamma_{h} / \Gamma_{h}[\%]$ & method \\
\hline \hline CLIC 380 & 0.38 & 1.0 & 4.7 & $\kappa$ \\
CLIC 1.5 & $0.38+1.5$ & 2.5 & 2.6 & $\kappa$ \\
CLIC 3.0 & $0.38+1.5+3$ & 5 & 2.5 & $\kappa$ \\
ILC 250 & 0.25 & 2.0 & 2.4 & EFT \\
ILC 500 & $0.25+0.5$ & $2.0+4.0$ & 1.6 & EFT \\
CEPC & 0.25 & 5.6 & 2.8 & $\kappa$ \\
FCC $_{\text {ee } 240}$ & 0.24 & 5.0 & 2.7 & $\kappa$ \\
FCC $_{\text {ee }}$ & $0.24+0.365$ & 6.5 & 1.3 & $\kappa$ \\
\hline \hline
\end{tabular}

where we used the fact that, within the framework discussed here, both $\sigma\left(e^{+} e^{-} \rightarrow Z h\right)$ and $\Gamma\left(h \rightarrow Z Z^{*}\right)$ are modified from their SM values by the same factor, $\kappa_{Z}^{2}$. The precision of this determination is then limited by the statistics of $h \rightarrow Z Z^{*}$ decays. In practice, for most colliders the $\kappa$-framework is used to extract the width parameter which benefits from additional measurements that are also sensitive to the width via similar reasoning. In particular, both the statistical precision and the model dependence can be reduced significantly if also $\sigma\left(e^{+} e^{-} \rightarrow h \nu_{e} \nu_{e}\right)$ and $\mathrm{BR}\left(h \rightarrow W W^{*}\right)$ as is possible for $\mathrm{FCC}_{e e}$ at $365 \mathrm{GeV}$, CLIC (at all proposed energies) and ILC at $500 \mathrm{GeV}$. This explains the substantial improvement observed when higher energy data are included. The estimated sensitivities of future $e^{+} e^{-}$colliders to the total width are given in Table III. It is much superior to the first and second determinations of the width at the LHC discussed above. A word of caution is, however, in place here. While the assumption that both the $Z h$ cross section and the $h \rightarrow Z Z$ decay width are modified by one simple factor, $\kappa_{Z}^{2}$, holds in the framework discussed in this subsection (extending the SM with a single light real singlet scalar), it is a modeldependent assumption, and cannot be assumed in general [17]. Thus, for ILC, the width has been extracted using an EFT fit with use of polarization and angular information instead. This is the reason that we quote also the method used in each experiment in Table III.

\section{B. $M_{s}>M_{h} / 2$}

The case of $M_{s}>M_{h} / 2$ is more challenging. In this case, there is a universal modification of all $h x x$ couplings, see Eq. (6). The partial width into any SM final state, $\Gamma(h \rightarrow f)$, as well as the total Higgs width, $\Gamma_{h}$, change by the same factor, $\left(1+\delta g_{h}\right)^{2}$. Consequently, the branching ratios into the various final states are unchanged from the SM, but the production rates are modified:

$$
\mu_{i}^{f} \equiv \frac{\sigma_{i}(p p \rightarrow h) \times \mathrm{BR}(h \rightarrow f)}{\left[\sigma_{i}(p p \rightarrow h) \times \mathrm{BR}(h \rightarrow f)\right]_{\mathrm{SM}}}=\left(1+\delta g_{h}\right)^{2} .
$$

Here $i$ is the Higgs production mode ( $\mathrm{ggF}, \mathrm{VBF}$, etc.). If there is no $Z_{2}$ symmetry, then doublet-singlet mixing is allowed, i.e. $\sin 2 \theta \neq 0$ (see Eq. (3)), and the dominant contribution to $\delta g_{h}$ in Eq. (6) is likely to come from the $(\cos \theta-1)$ term, and consequently

$$
\mu_{i}^{f} \approx \cos ^{2} \theta
$$

If there is an unbroken $Z_{2}$ symmetry,

$$
\left(\mu_{i}^{f}\right)^{\left(Z_{2}\right)}=1-\frac{\left|\lambda_{h s}\right|^{2} v^{2}}{8 \pi^{2}} I_{B}\left(M_{h}^{2} ; M_{s}^{2}, M_{s}^{2}\right) .
$$

For $M_{s}^{2} \gg M_{h}^{2}$, we have

$$
\left(\mu_{i}^{f}\right)^{\left(Z_{2}\right)}\left(M_{s}^{2} \gg M_{h}^{2}\right) \approx 1+\frac{\left|\lambda_{h s}\right|^{2}}{48 \pi^{2}} \frac{v^{2}}{M_{s}^{2}} .
$$


Table IV: Precision on the dominant Higgs production cross sections for the following $e^{+} e^{-}$colliders: CLIC [16], CEPC [7], ILC [17] and $\mathrm{FCC}_{\mathrm{ee}}$.

\begin{tabular}{|c|c|c||c|}
\hline \hline Collider & $\sqrt{s}[\mathrm{TeV}]$ & $\mathcal{L}\left[\mathrm{ab}^{-1}\right]$ & $\delta \sigma_{Z h} / \sigma_{Z h}[\%]$ \\
\hline \hline CLIC & 0.38 & 1.0 & 1.3 \\
ILC & 0.25 & 2.0 & 0.7 \\
CEPC & 0.25 & 5.6 & 0.5 \\
FCC $_{\mathrm{ee}}$ & 0.24 & 5.0 & 0.5 \\
\hline \hline
\end{tabular}

Under the assumption that the values of the signal strengths $\mu_{i}^{f}$ are the same for all production processes $i$ and decay channels $f$, a fit to the ATLAS and CMS data at $\sqrt{s}=7$ and $8 \mathrm{TeV}$, with $\mu$ as the parameter of interest, results in the best fit value [28]:

$$
\mu=1.09_{-0.10}^{+0.11}
$$

With the full HL-LHC dataset, the LHC now projects, for the two best measured production rates, $1.6 \%$ for the ggF channel and $3.1 \%$ for the VBF channel. There is still a question of theory uncertainties on the ggF and VBF cross sections. One can argue that the theory precision on $\mathrm{VBF}$ will be $\lesssim 1 \%$ while for ggF this is harder to estimate. In principle the above numbers already fold in acceptance uncertainties due to theory but not yet overall normalization uncertainties.

As concerns the ILC, Ref. [17] (Table 6) estimates the accuracy on $\sigma_{Z h}$ to be $0.7 \%$, while for CEPC and FCC a precision of $0.5 \%$ is expected. The accuracy on the measurement of $\sigma_{Z h}$ in various proposed $e^{+} e^{-}$colliders is presented in Table IV.

A particularly interesting issue in which the addition of a singlet scalar to the SM is relevant is the possibility that its coupling to the Higgs field makes the EWPT first order. Ref. [29] obtains that the lower bound on $\left|\lambda_{h s}\right|^{2} / M_{s}^{2}$ is such that

$$
\mu_{i}^{f}-1 \gtrsim 0.6 \%
$$

We conclude that the ILC, CEPC and $\mathrm{FCC}_{e e}$ may be able to lend support or exclude this scenario as they reach a precision of $0.5-0.7 \%$. However, the sensitivity may only be at the level of one standard deviation for all three colliders if the deviation is near its lower bound.

At CLIC, this scenario has been studied in detail [73] and it was estimated that, based on the Higgs coupling measurements, a constraint of

$$
\mu_{i}^{f}-1 \lesssim 0.24 \% \text { at } 95 \% \text { C.L. }
$$

can be derived when including all stages, making it sensitive to probing the order of the EWPT.

The width measurements presented in Table III are also sensitive to this scenario but the precision is inferior than that of the total cross sections.

\section{WHAT IS THE SOLUTION OF THE FLAVOR PUZZLE(S)?}

There are several reasons that make the study of flavor physics via Higgs physics well motivated. First, flavor physics raises three puzzles [30]:

- The Standard Model flavor puzzle: Why is there structure (smallness and hierarchy) in the charged fermion masses and the CKM mixing angles?

- The neutrino flavor puzzle: Why, in contrast to the charged fermions, there seems to be no structure (neither hierarchy nor degeneracy nor smallness) in the neutrino-related flavor parameters?

- The new physics flavor puzzle: If there is new physics at the TeV scale, what is the mechanism (alignment and/or degeneracy) that prevents it from significantly modifying the SM predictions for flavor changing neutral current processes? 
Various models have been suggested to answer one or more of these questions. The best hope to make further progress is by measuring new flavor parameters (beyond the matrix elements of the CKM matrix). Measurements of the Yukawa couplings of $h$ provide such an opportunity.

Second, within the Standard Model (SM), flavor changing neutral current (FCNC) processes are suppressed by three factors:

- Loop suppression;

- CKM suppression;

- GIM suppression.

This unique situation allows the measurements of FCNC processes to probe new physics at very high scales. The search for Higgs-related flavor violating processes, such as $t \rightarrow c h$ or $h \rightarrow \tau \mu$, provides a new arena for FCNC measurements.

Within the SM, there is a clear prediction for the tree-level values of the Yukawa couplings:

$$
Y_{F}=(\sqrt{2} / v) M_{F} \quad(F=U, D, E) .
$$

This relation between the Yukawa matrices and the mass matrices encompasses, in fact, three features:

- Proportionality: The diagonal Yukawa couplings $\left(y_{f} \equiv Y_{f f}\right)$ are proportional to the corresponding masses. For example,

$$
y_{\mu} / y_{\tau}=m_{\mu} / m_{\tau}
$$

- Factor of proportionality: The factor that connects the mass to the diagonal Yukawa coupling is $\sqrt{2} / v$. For example,

$$
y_{\tau}=(\sqrt{2} / v) m_{\tau}
$$

- Diagonality: The off-diagonal Yukawa couplings vanish. For example,

$$
Y_{\mu \tau}=0
$$

The three examples in Eqs. (24), (25) and (26) demonstrate that, by measuring (or constraining) the rates of $h \rightarrow \tau \tau, h \rightarrow \mu \mu$ and $h \rightarrow \tau \mu$, we can test each of the three features predicted by the SM separately. In case that deviations from the SM predictions are established, the pattern of deviations will allow us to distinguish between various frameworks that aim to solve the flavor puzzles. This is demonstrated in Table V, where the predictions of various flavor models for the Yukawa couplings are given (adapted from [31]). In this Table, the scale $\Lambda$ is the scale that suppresses dimension-six terms in the SM effective-field-theory (SM-EFT) of the form

$$
\frac{z_{i j}}{\Lambda^{2}}\left(\Phi^{\dagger} \Phi\right) \overline{L_{i}} \tilde{\Phi} E_{j}
$$

where $L_{i}$ are the lepton doublet fields in the $(1,2)_{-1 / 2}$ representation, and $E_{j}$ are the lepton singlet fields in the $(1,1)_{-1}$ representation.

In Table VI, we present the predictions in various theoretical models for the flavor changing $t \rightarrow h q$ decays. The numerical estimates for the SM, 2HDM, MSSM and RS frameworks are taken from Ref. [32] (Table 1-7). The parametric suppression factors in the SM-EFT subject to minimal flavor violation (MFV) or to Froggatt-Nielsen (FN) selection rules are taken from Refs. [33] and [34], respectively.

In the following, the current measurements and expected accuracy of future measurements for the parameters relevant to flavor are discussed. For this purpose, we assume the future collider parameters presented in Section II.

The present status of the measured $y_{f}$ values is presented in Table VII, and its compatibility with the SM prediction of proportionality is presented in Fig. 1. For the various colliders, different methods are used to extract the couplings, leading to some model-dependence at the level of $(0.5-1.0) \%$. For the third generation fermions the current LHC precision is about $(10-25) \%$. With HL-LHC it will be improved to $(2-4) \%$. The other future machines will improve the precision by another factor of $\sim 3$.

For the second (and first) generation fermions currently only upper limits are available. As concerns $y_{\mu}$, the HL-LHC will measure it to better than $5 \%$, and future $e^{+} e^{-}$colliders are not expected to improve this further significantly. $\mathrm{FCC}_{h h}$ will be able to measure the ratio $\mathrm{BR}(h \rightarrow \mu \mu) / B R(h \rightarrow 4 \mu)$ with a precision of $1.3 \%$. As concerns $y_{c}$, 
Table V: Predictions for di-lepton Higgs decays in various flavor models: SM, natural flavor conservation (NFC), minimal supersymmetric SM (MSSM), minimal flavor violation (MFV), Froggatt-Nielsen (FN) models and Giudice-Lebedev (GL). (See [31] for details.)

\begin{tabular}{cccc}
\hline \hline Model & $\frac{y_{\tau}}{\sqrt{2} m_{\tau} / v}$ & $\frac{y_{\mu} / y_{\tau}}{m_{\mu} / m_{\tau}}$ & $\frac{\mathrm{BR}(h \rightarrow \mu \tau)}{\mathrm{BR}(h \rightarrow \tau \tau)}$ \\
\hline SM & 1 & 1 & 0 \\
NFC & $V_{h \ell}^{*} v / v_{\ell}$ & 1 & 0 \\
MSSM & $\sin \alpha / \cos \beta$ & 1 & 0 \\
MFV & $1+\mathcal{O}\left(v^{2} / \Lambda^{2}\right)$ & $1+\mathcal{O}\left(m_{\tau}^{2} / \Lambda^{2}\right)$ & 0 \\
FN & $1+\mathcal{O}\left(v^{2} / \Lambda^{2}\right)$ & $1+\mathcal{O}\left(v^{2} / \Lambda^{2}\right)$ & $\mathcal{O}\left(\left|U_{23}\right|^{2} v^{4} / \Lambda^{4}\right)$ \\
GL & 3 & $5 / 3$ & $\mathcal{O}\left[(25 / 9)\left(m_{\mu}^{2} / m_{\tau}^{2}\right)\right]$ \\
\hline \hline
\end{tabular}

Table VI: Predictions $\operatorname{BR}(t \rightarrow h q)(q=u, c)$ in various models. (See [32-34] for details.)

\begin{tabular}{|c|c|c|c|c|c|c|}
\hline & SM & $2 \mathrm{HDM}$ & MSSM & RS & SM-EFT(MFV) & SM-EFT $(\mathrm{FN})$ \\
\hline$t \rightarrow h c$ & $3 \times 10^{-15}$ & $2 \times 10^{-3}$ & $\leq 10^{-5}$ & $\leq 10^{-4}$ & $y_{b}^{4}\left(V_{c b} V_{t b}\right)^{2}(v / \Lambda)^{4}$ & $\left|V_{c b}\right|^{2}(v / \Lambda)^{4}$ \\
\hline$t \rightarrow h u$ & $2 \times 10^{-17}$ & $6 \times 10^{-6}$ & $\leq 10^{-5}$ & - & $y_{b}^{4}\left(V_{u b} V_{t b}\right)^{2}(v / \Lambda)^{4}$ & $\left|V_{u b}\right|^{2}(v / \Lambda)^{4}$ \\
\hline
\end{tabular}

the HL-LHC expects to be sensitive to values 6-21 times larger than the SM value. Future colliders will probe it in earnest with a precision of (1-2)\%. At hadron colliders it is expected that very large anomalous values of the strange quark coupling can be probed on $\phi \gamma$ decays [27]: for HL-LHC (FCC) the sensitivity is $-35<y_{s} / y_{b}^{\mathrm{SM}}<57$ $\left(-2<y_{s} / y_{b}^{\mathrm{SM}}<24\right)$. At $e^{+} e^{-}$colliders values of $\mathcal{O}(10) \times \mathrm{SM}$ rates might be in reach [38]. As concerns the first generation, the best hope for probing the SM value of $y_{e}$ directly is to run one of the circular $e^{+} e^{-}$colliders at $\sqrt{s} \approx m_{h}$ for several years. For the up and down quarks the projected sensitivity of the HL-LHC is more than 1000 times the SM value, and is only obtained indirectly.

The GL model (see Table V) is already excluded by the current data presented in Table VII (by both the $y_{\tau}$ measurement and the $y_{\mu}$ upper limit). For the FN model, the current constraints imply a lower bound on the scale of new physics, $\Lambda / \sqrt{z_{\tau \tau}} \gtrsim 600 \mathrm{GeV}$. The expected precision of HL-LHC will increase the reach to $\Lambda / \sqrt{z_{\tau \tau}} \sim 2 \mathrm{TeV}$, while some of the future facilities can reach up to about $\Lambda / \sqrt{z_{\tau \tau}} \sim 4 \mathrm{TeV}$.

The present status of measurements and the future projections for off-diagonal branching ratios are given in Table VIII. At present most upper limits are between $0.1 \%$ and $1 \%$. With HL-LHC they will be improved by an order of magnitude to about $1 \times 10^{-4}$. For the $t \rightarrow q h$ decays, it can be expected that the sensitivity of future $e^{+} e^{-}$colliders is to branching ratios of order $10^{-5}$, similar to other rare decay modes of the top quark [8], but no dedicated study is available. With FCC-hh an improvement by another 1-2 orders of magnitude is expected, depending on the systematic uncertainties that will be obtained. These values are somewhat interesting in view of theories beyond the SM, see Table VI.

Finally, let us mention that, while the third generation couplings are already constrained to be within about $10 \%$ of the SM values, large deviations might still arise in measurements of the Yukawa couplings of the first two generations and of flavor changing decays. The experimental effort to search for these decays has thus the potential to make surprising discoveries, even if the sensitivity is far from the SM predictions.

\section{ADDITIONAL QUESTIONS}

\section{A. Is $h$ alone?}

Within the SM, there is a single real scalar field, the Higgs boson $h_{\mathrm{SM}}$. It originates from a field $\Phi(1,2)_{1 / 2}$ that is a doublet of the $S U(2)$ gauge symmetry. In the unitary gauge, the Higgs field is given by

$$
\Phi=\left(\begin{array}{c}
0 \\
\frac{1}{\sqrt{2}}\left(v+h_{\mathrm{SM}}\right)
\end{array}\right) .
$$


Table VII: Experimental status and future projections of the diagonal Yukawa couplings $y_{f}$, and the accuracy estimated for future experiments in \%. The accuracy quoted for future experiments is based on combining those data with the HL-LHC, except for CLIC and FCC240 where only the accuracy of the future collider is stated. Upper bounds are given at 95\% confidence level. The first line in each row shows the CMS result and the second line the ATLAS result. In both cases, a BSM contribution is allowed, the $\gamma \gamma$ and $g g$ loop processes are treated with effective couplings, and the $Z \gamma$ process is resolved. For the $e^{+} e^{-}$ colliders, the same assumptions on the loops are made when using the so-called $\kappa$-framework. For LHC and HL-LHC [27] , $\mathrm{BR}_{\mathrm{BSM}}=0$ is assumed. For ILC an EFT fit is used to extract the values and the values are combined with HL-LHC [6]. For all other colliders the $\kappa$-fit results are quoted from Refs, $[7,8,37,39]$ and no combination with HL-LHC is made. All upper limits are given at $95 \%$ C.L.. For the FCC-ee, an upper limit of 1.6 can be set on $y_{e} / y_{e}^{\mathrm{SM}}$ if one year of running at $\sqrt{s} \approx m_{h}$ is performed. When no value is available in the literature, a - is shown.

\begin{tabular}{|c|c|c|c|c|c|c|c|c|c|c|}
\hline Observable & Current range & HL-LHC & LC250 & $\mathrm{ILC} 250+500$ & $\begin{array}{r}\text { CLIC3 } \\
\text { c }\end{array}$ & $\begin{array}{l}\mathrm{IC} 30 \\
\%)\end{array}$ & CEPC & $\mathrm{CC} 2$ & FCC365 & LHeC \\
\hline$y_{t} / y_{t}^{\mathrm{SM}}$ & $\begin{array}{l}1.02_{-0.15}^{+0.19}[35] \\
1.05_{-0.13}^{+0.14}[36]\end{array}$ & 3.4 & - & 6.3 & - & 2.9 & - & - & - & - \\
\hline$y_{b} / y_{b}^{\mathrm{SM}}$ & $\begin{array}{l}0.91_{-0.16}^{+0.17}[35] \\
0.85_{-0.14}^{+0.13}[36] \\
\end{array}$ & 3.7 & 1.0 & 0.60 & 1.3 & 0.2 & 1.0 & 1.4 & 0.67 & 1.1 \\
\hline$y_{\tau} / y_{\tau}^{\mathrm{SM}}$ & $\begin{array}{l}0.93 \pm 0.13[35] \\
0.95 \pm 0.13[36]\end{array}$ & 1.9 & 1.2 & 0.77 & 2.7 & 0.9 & 1.2 & 1.4 & 0.78 & 1.3 \\
\hline$y_{c} / y_{c}^{\mathrm{SM}}$ & $<6.2[40,41]$ & $<220$ & 1.8 & 1.2 & 4.1 & 1.3 & 1.9 & 1.8 & 1.2 & 3.6 \\
\hline$y_{\mu} / y_{\mu}^{\mathrm{SM}}$ & $\begin{array}{c}0.72_{-0.72}^{+0.50}[35] \\
<1.63[36]\end{array}$ & 4.3 & 4.0 & 3.8 & - & 5.6 & 5.0 & 9.6 & 3.4 & - \\
\hline$y_{e} / y_{e}^{\mathrm{SM}}$ & $<611[42]$ & - & - & & - & - & - & - & $<1.6^{(+)}$ & - \\
\hline
\end{tabular}

Table VIII: Experimental status of measurements that depend on off-diagonal Yukawa couplings (assuming SM production rates). The LHC $t \rightarrow q h$ measurements are based on $36.1 \mathrm{fb}^{-1}$. For $\mathrm{FCC}_{h h}$ the range quoted for $t \rightarrow q h$ reflects the size of the systematic uncertainty on the background of $(0-5) \%$.

\begin{tabular}{lccc}
\hline \hline Observable & Upper bound & HL-LHC & FCC-hh \\
\hline $\operatorname{BR}(t \rightarrow c h)$ & $1.1 \times 10^{-3}[43]$ & $10^{-4}[44]$ & $(4.9-16) \times 10^{-6}[8]$ \\
$\operatorname{BR}(t \rightarrow u h)$ & $1.2 \times 10^{-3}[43]$ & $10^{-5}[44]$ & $(2.5-28) \times 10^{-6}[8]$ \\
$\operatorname{BR}(h \rightarrow \tau \mu)$ & $2.5 \times 10^{-3}[45,46]$ & $5 \times 10^{-4}[27]$ & - \\
$\operatorname{BR}(h \rightarrow \tau e)$ & $6.1 \times 10^{-3}[45,46]$ & $5 \times 10^{-4}[20]$ & - \\
$\operatorname{BR}(h \rightarrow \mu e)$ & $3.4 \times 10^{-4}[47]$ & - & - \\
\hline \hline
\end{tabular}

The scalar $h$ discovered in the ATLAS and CMS experiments [1,2] has measured properties that are all consistent, within present experimental accuracy, with the SM predictions for the Higgs boson [28]. The question that is being asked is whether the observed Higgs boson is the SM Higgs boson. In fact, one can separate this question to two:

1. Does $h$ originate from purely a doublet?

2. If there is more than a single doublet, is $h$ in exactly the direction of $v$ ?

As concerns the first question, an example of a scenario where $h$ does not originate from purely a doublet is that of doublet-singlet mixing, discussed in Section III. As concerns the second question, we briefly discuss in this subsection the scenario of two Higgs doublet models (2HDM).

In a 2HDM with Natural Flavor Conservation (NFC), there are three well defined bases:

1. The NFC basis $\left(\Phi_{1}, \Phi_{2}\right)$, where each fermionic sector couples to either $\Phi_{1}$ or $\Phi_{2}$, but not to both.

2. The Higgs basis $\left(\Phi_{v}, \Phi_{A}\right)$, where $\left\langle\Phi_{v}\right\rangle=v / \sqrt{2}$ and $\left\langle\Phi_{A}\right\rangle=0$.

3. The mass basis $\left(\Phi_{h}, \Phi_{H}\right)$, which is the mass basis for the CP-even neutral scalars $h$ and $H$. 


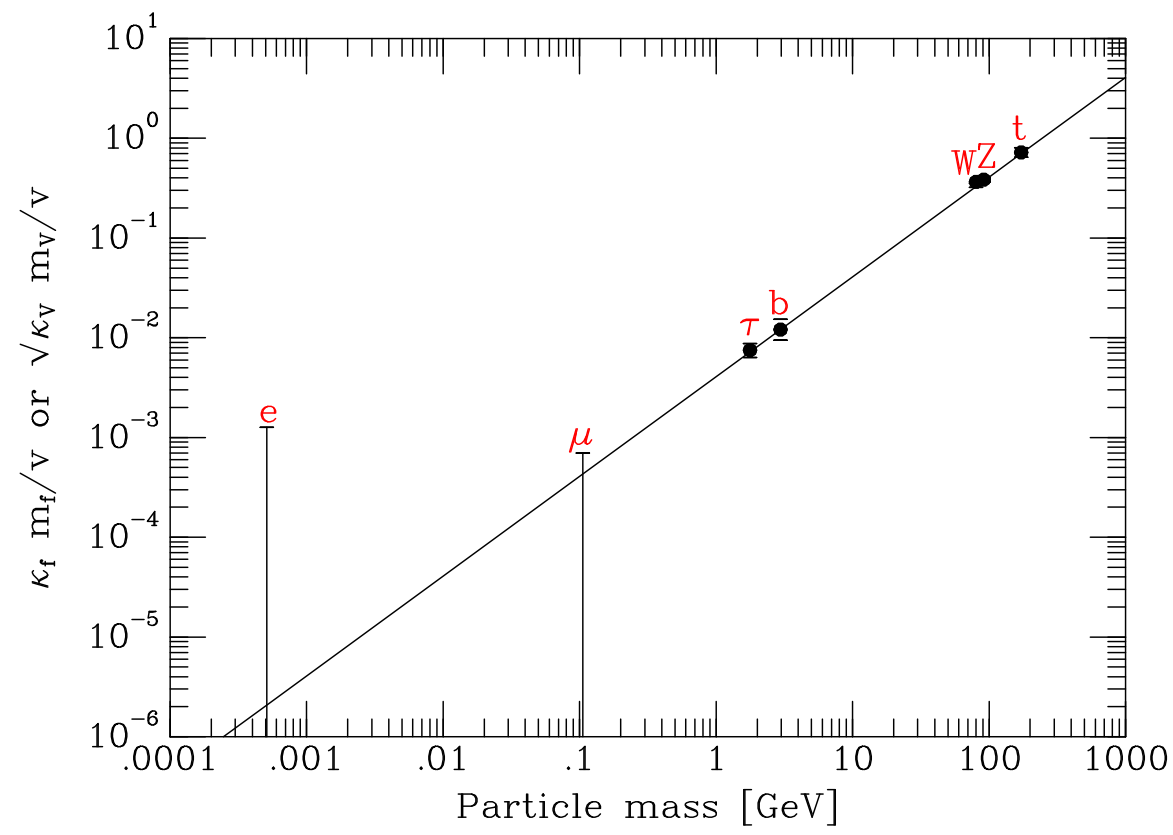

Figure 1: The currently allowed experimental ranges for the Higgs couplings. For the Yukawa couplings, we present $\kappa_{f} m_{f} / v$. For the coupling to the electroweak vector bosons, we present $\sqrt{\kappa_{V}} m_{V} / v$. The SM prediction is presented by the diagonal solid line. (Keith Ellis, private communication.)

The rotation angle from the $\left(\Phi_{1}, \Phi_{2}\right)$ to the $\left(\Phi_{v}, \Phi_{A}\right)\left[\left(\Phi_{h}, \Phi_{H}\right)\right]$ basis is denoted $\beta[\alpha]$. We define $\kappa_{x}$ via

$$
\kappa_{x} \equiv \frac{g_{h x x}}{g_{h x x}^{\mathrm{SM}}}
$$

In all 2 HDMs we have $(V=W, Z)$

$$
\begin{aligned}
& \kappa_{V}=\sin (\beta-\alpha) \simeq 1-\frac{1}{2} \cos ^{2}(\beta-\alpha), \\
& \kappa_{h} \simeq 1-\frac{2 m_{A}^{2}}{m_{h}^{2}} \cos ^{2}(\beta-\alpha) .
\end{aligned}
$$

Note that for $m_{A}^{2} \gg m_{h}^{2}$, we have $\left(m_{A}^{2} / m_{h}^{2}\right) \cos ^{2}(\beta-\alpha) \simeq \cos (\beta-\alpha)$. In all NFC models, where by definition $\Phi_{1}$ is the doublet that does not couple to the up sector,

$$
\begin{aligned}
& \kappa_{t}=\sin (\beta-\alpha)+\frac{\cos (\beta-\alpha)}{\tan \beta}, \\
& \kappa_{b}=\sin (\beta-\alpha)-\cos (\beta-\alpha) \tan \beta \text { or } \kappa_{t}, \\
& \kappa_{\tau}=\sin (\beta-\alpha)-\cos (\beta-\alpha) \tan \beta \text { or } \kappa_{t},
\end{aligned}
$$

where the two options on the RHS of $\kappa_{b}$ correspond to the cases where $b$ couples to $\Phi_{1}$ or $\Phi_{2}$, respectively, and similarly for $\kappa_{\tau}$. The largest deviations in these models arise in the down or charged lepton sector, if either or both couple to the doublet to which the up sector does not couple. If all fermions couple to $\Phi_{2}$, then the largest deviation is expected in $\lambda_{h}$ [48]. At tree level, the results for the MSSM are the same as those of a type II 2 HDM.

We conclude that the most promising precision measurements of Higgs decays for obtaining hints of $2 \mathrm{HDM}$ are those of $h \rightarrow b \bar{b}$ and $h \rightarrow \tau^{+} \tau^{-}$. The future projections of measuring $y_{b}$ and $y_{\tau}$ in future experiments can be read off Table VII. An even more powerful probe of $2 \mathrm{HDM}$ is the combination of these two couplings with a measurement of $\kappa_{V}$, see Table IV. With $\kappa_{Z}=\sin (\beta-\alpha)$ measured very accurately, an accuracy of $\mathcal{O}(1 \%)$ on $y_{\tau}$ will essentially constrain $\cos (\beta-\alpha) \tan \beta$ at that level in NFC models where the $\tau$ couples to $\Phi_{1}$ only.

\section{B. Is $h$ elementary?}

The question of Higgs compositeness is interesting in its own sake, but also in the context of mechanisms to explain the smallness of $m_{h}^{2}$ compared to $m_{\mathrm{Pl}}^{2}$. Various models have been proposed where the Higgs is a pseudo-Goldstone 
boson which emerges from a strongly interesting sector [49-51]. An effective field theory framework for the strongly interacting light Higgs (SILH) incorporates the basic features of a large class of these models [52]. The low energy effective SILH Lagrangian depends on essentially two parameters: $m_{\rho}$, the mass scales of new resonances emerging from the strongly interacting sector, and $g_{\rho}$, their coupling. It is useful to define the dimensionless combination

$$
\xi=g_{\rho}^{2} v^{2} / m_{\rho}^{2}
$$

The terms in the SILH Lagrangian that are relevant to our purposes are given by

$$
\mathcal{L}_{\mathrm{SILH}}=\frac{c_{H} \xi}{2 v^{2}} \partial^{\mu}\left(\phi^{\dagger} \phi\right) \partial_{\mu}\left(\phi^{\dagger} \phi\right)+\frac{c_{y} \xi y_{f}}{v^{2}} \phi^{\dagger} \phi \overline{f_{L}} \phi f_{R}+\text { h.c.. }
$$

Naive dimensional analysis (NDA) suggests that $c_{H}$ and $c_{y}$ are $\mathcal{O}(1)$. The theoretical framework only allows the parameters to be in the range

$$
1 \leq g_{\rho} \leq 4 \pi, \quad \xi \leq 1
$$

Electroweak precision measurements give stronger constraints. The new physics contribution to $\hat{S}$ and $\hat{T}$ can be parameterized as follows [53]:

$$
\begin{aligned}
& \Delta \hat{S}=\frac{g^{2}}{96 \pi^{2}} \xi\left[\log \left(\frac{\Lambda}{m_{h}}\right)+6 \alpha\right]+\frac{m_{W}^{2}}{m_{\rho}^{2}}, \\
& \Delta \hat{T}=-\frac{g^{\prime 2}}{32 \pi^{2}} \xi\left[\log \left(\frac{\Lambda}{m_{h}}\right)-6 \beta \frac{y_{t}^{2}}{g^{\prime 2}}\right],
\end{aligned}
$$

where $\Lambda=4 \pi m_{\rho} / g_{\rho}$ and the coefficients $\alpha, \beta=\mathcal{O}(1)$ and could have either sign. The resulting constraints read [53]

$$
\xi \leq 0.15, \quad m_{\rho} \geq 3 \mathrm{TeV}
$$

when marginalizing over $\alpha$ and $\beta$ (or $\xi \leq 0.025$ and $m_{\rho} \geq 4 \mathrm{TeV}$ for $\alpha=\beta=0$ ).

The modifications of the Higgs couplings are given by

$$
\begin{aligned}
\Delta g_{V} / g_{V}^{\mathrm{SM}} & =-c_{H} \xi / 2, \\
\Delta g_{f} / g_{f}^{\mathrm{SM}} & =-c_{H} \xi / 2-c_{y} \xi, \\
\Delta g_{g} / g_{g}^{\mathrm{SM}} & =-c_{H} \xi / 2-c_{y} \xi, \\
\Delta g_{\gamma} / g_{\gamma}^{\mathrm{SM}} & =-c_{H} \xi / 2+0.3 c_{y} \xi .
\end{aligned}
$$

Thus, the maximal possible deviations are [54]

$$
\begin{aligned}
& \Delta g_{V} / g_{V}^{\mathrm{SM}} \approx-0.08 c_{H}, \\
& \Delta g_{f} / g_{f}^{\mathrm{SM}}=\Delta g_{g} / g_{g}^{\mathrm{SM}} \approx-0.08-0.15 \frac{c_{y}}{c_{H}} \\
& \Delta g_{\gamma} / g_{\gamma}^{\mathrm{SM}} \approx-0.08+0.05 \frac{c_{y}}{c_{H}} .
\end{aligned}
$$

E.g. for $c_{H}=c_{y}=1$ the maximal deviations are $-0.08,-0.20$ and -0.03 for the $g_{V}, g_{f, g}$ and $g_{\gamma}$ couplings, respectively. The estimates of the reach on $c_{H} \xi$ of Higgs-related measurements for various colliders are collected in Table IX. Comparing this to the maximal possible deviations given above, it is clear that future colliders will probe this aspect in a very interesting regime. Currently the precision obtained by ATLAS and CMS on $\kappa_{Z}$ and $\kappa_{W}$ is about $10 \%$ each $[35,36]$, resulting in a sensitivity of about $20 \%$ on $c_{H} \xi$. Ref. [55] employs a more general framework, based on nonlinear shift symmetries acting on $h$ and assuming custodial symmetry in the strong sector, and quotes the presently allowed $68 \%$ CL range as

$$
\xi=-0.041_{-0.094}^{+0.090}
$$

Ref. [53] compared the sensitivity of the Higgs related measurements and the direct resonance searches at (and beyond) the LHC within the framework of composite Higgs models. They reach the conclusion that the Higgs related measurements will be more sensitive for large $g_{\rho}$. Specifically, the indirect measurements explore novel territory for $g_{\rho} \gtrsim 4.5$. 
Table IX: Summary of the reach on $\xi$ for various collider options, based on Ref. [53] but updated using the recent projections on the $\kappa_{V}$ sensitivity. See discussion in Section VB.

\begin{tabular}{ccccc}
\hline \hline Collider & Energy & Luminosity & $\Delta g_{V} / g_{V}(\%)\left(c_{H} \xi\right)_{\max }[1 \sigma]$ \\
\hline \hline HL-LHC & $14 \mathrm{TeV}$ & $3 \mathrm{ab}^{-1}$ & 1.3 & 0.026 \\
\hline ILC & $250 \mathrm{GeV}$ & $2 \mathrm{ab}^{-1}$ & 0.56 & 0.011 \\
& $+500 \mathrm{GeV}$ & $4 \mathrm{ab}^{-1}$ & 0.38 & 0.008 \\
\hline CLIC & $380 \mathrm{GeV}$ & $1 \mathrm{ab}^{-1}$ & 0.6 & 0.012 \\
& $+1.4 \mathrm{TeV}$ & $2.5 \mathrm{ab}^{-1}$ & 0.6 & 0.012 \\
& $+3.0 \mathrm{TeV}$ & $5 \mathrm{ab}^{-1}$ & 0.6 & 0.012 \\
\hline FCC $_{e e}$ & $240 \mathrm{GeV}$ & $2 \times 5.0 \mathrm{ab}^{-1}$ & 0.2 & 0.004 \\
& $+350 \mathrm{GeV}$ & $2.6 \mathrm{ab}^{-1}$ & 0.17 & 0.003 \\
\hline $\mathrm{CEPC}^{*}$ & $240 \mathrm{GeV}$ & $2 \times 5.6 \mathrm{ab}^{-1}$ & 0.25 & 0.005 \\
\hline \hline
\end{tabular}

\section{What keeps $m_{h}^{2} \ll m_{\mathrm{Pl}}^{2}$ ?}

If the SM is only a low energy effective theory, and at some high scale $\Lambda_{\mathrm{NP}}$ there are new degrees of freedom which couple to the Higgs boson, then the mass-squared of the Higgs boson get corrections of $\mathcal{O}\left(\Lambda_{\mathrm{NP}}^{2}\right)$, and a fine-tuned cancelation with the bare $\mu^{2}$ term is needed to keep the Higgs mass light. Possible solutions to this fine-tuning problem can be classified to symmetry-related mechanisms, and dynamical scenarios.

The two most intensively studied frameworks where the solution is symmetry related are composite Higgs and Supersymmetry. We discussed the composite Higgs framework in Section V B.

As concerns the Higgs couplings in the minimal supersymmetric SM (MSSM), the situation is similar to the 2HDM discussed in Section VA with NFC type II, and with some additional constraints arising from relations to processes involving the supersymmetric partners of the SM degrees of freedom. Ref. [54] obtained the maximal possible deviations of the $h$ couplings, assuming that the scalar degrees of freedom of the second Higgs doublet are too heavy to be directly observed at the LHC. They obtain

$$
\begin{aligned}
1-\kappa_{V} & \lesssim 0.01 \\
1-\kappa_{t} & \lesssim 0.03 \\
1-\kappa_{b} & \lesssim 0.1-1 .
\end{aligned}
$$

A dynamical solution to the fine tuning problem is provided by relaxion models [56]. The Higgs mass depends on a time-dependent VEV of a scalar field which rolls until it stops at a value much smaller than the cut-off scale of the theory. Unlike models of composite Higgs or Supersymmetry, where many new degrees of freedom are predicted at a scale not much higher than the electroweak symmetry breaking scale, in this framework there is a single new scalar, SM-singlet, degree of freedom, the relaxion $\phi_{R}$. Much of the relevant phenomenology comes from the generic situation where the relaxion and the Higgs mix [19]. Thus, the relaxion inherits the Higgs couplings, suppressed by the small $\phi_{R}-h$ mixing angle. This scenario was discussed in Section III, particularly in the case of $M_{s}<M_{h} / 2$.

\section{Was the electroweak phase transition (strongly) first order?}

At a temperature of order $100 \mathrm{GeV}$, the Universe went through a transition from a high temperature symmetric phase $(\langle\Phi\rangle=0)$ to a state with broken electroweak symmetry $(\langle\Phi\rangle=v / \sqrt{2} \neq 0)$. If the SM is a good low energy effective theory up to the $\mathrm{TeV}$ scale, then the electroweak phase transition (EWPT) is a crossover transition. If, on the other hand, the EWPT was first-order, then electroweak baryogenesis was possible. In fact, successful EWBG requires that the phase transition is strongly first order,

$$
\frac{v\left(T_{c}\right)}{T_{c}} \gtrsim 1
$$

where $T_{c}$ is the temperature at which the phase transition takes place. This requirement usually implies new degrees of freedom that are not much heavier than the EW scale and which couple to the Higgs field with a coupling that is not small. 
The new physics that can make a first-order EWPT can be broadly classified to four classes [57], distinguished by the physics that is responsible for generating the barrier between the EW-symmetric and EW-breaking minima:

- Thermally driven. New bosonic degrees of freedom generate a finite-temperature term in the effective potential of the form $-T\left(h^{2}\right)^{3 / 2}$.

- Tree-level renormalizable operators. Additional scalars, typically $S U(2)_{L}$-singlets or doublets, mix with the Higgs boson and generate an effective, temperature-independent, $\phi^{3}$ term.

- Tree level non-renormalizable operators. The most intensively studied example is a dimension-six term $\left(\Phi^{\dagger} \Phi\right)^{3}$ (with a negative $\lambda$ for the $\lambda\left(\Phi^{\dagger} \Phi\right)^{2}$ term).

- Loop corrections. The most intensively studied example is an effective term of the form $\kappa h^{4} \ln \left(h^{2} / M^{2}\right)$ in the Higgs potential.

Loops involving scalar particles induce a cubic term in the effective potential at high temperature that can modify the EWPT into a first-order one (see, for example, Ref. [29]). Consider then a new scalar $S$, with coupling to the Higgs field $\Phi$ :

$$
V_{S \Phi}=\kappa|S|^{2}|\Phi|^{2}
$$

To make the phase transition first-order, we need $\kappa=\mathcal{O}(1)$ and $m_{S} \lesssim 400 \mathrm{GeV}$. This term modifies the following Higgs observables:

1. If $S$ is colored, the $h g g$ coupling is modified. Thus, at hadron colliders, a measurement of $\mu_{\mathrm{ggF}}$ is best suited to probe this scenario, which is expected to have a precision of about $2 \%$ at HL-LHC. At lepton colliders, the best observable is $\operatorname{BR}(h \rightarrow g g)$ which can be probed with a precision of about 1.5-2.5\%.

2. If $S$ is electromagnetically charged, the $h \gamma \gamma$ coupling is modified. Thus, a measurement of $\mathrm{BR}(h \rightarrow \gamma \gamma)$ is best suited to probe this scenario. At HL-LHC a precision of about $2.6 \%$ is expected, and lepton colliders are not expected to improve this significantly. A substantial improvement is expected from $\mathrm{FCC}_{h h}$ with about $0.5 \%$.

3. Independently of the quantum numbers of $S$, the $h Z Z$ coupling will be modified, since $S$ renormalizes the Higgs wavefunction [10,58]. The case where $S$ is neither colored nor electromagnetically charged was discussed in Section III.

Models of strongly first-order EWPT due to renormalizable tree-level operators will be further tested by Higgs measurements of the invisible Higgs decays or by the bounds on the mixing with singlet scalars, discussed in Section III, and by various other measurements (see e.g. [59, 60]). For models of strongly first-order EWPT due to nonrenormalizable tree-level operators,

$$
V=\mu^{2}\left(\Phi^{\dagger} \Phi\right)+\lambda\left(\Phi^{\dagger} \Phi\right)^{2}+\frac{\rho}{\Lambda^{2}}\left(\Phi^{\dagger} \Phi\right)^{3}
$$

the main consequence is a modification of the relation between the Higgs mass, the VEV and the trilinear Higgs self-coupling:

$$
\lambda_{h h h}=3 \frac{m_{h}^{2}}{v}+6 \rho \frac{v^{3}}{\Lambda^{2}} .
$$

The required size of $\rho v^{2} / \Lambda^{2}$ is such that deviation of $\mathcal{O}(1)$ in $\lambda_{h h h}$ are predicted. Table $\mathrm{X}$ summarizes the expected sensitivity of various future colliders.

\section{E. Do CP violating $h$ interactions generate the baryon asymmetry?}

Complex Yukawa couplings could play a CP violating role in electroweak baryogenesis, see e.g. Refs. [61-64]. Various suggestions of how to measure such CP violating Higgs interactions have been made, in particular for $h \tau \tau$ and $h t t$ couplings, see e.g. Refs. [65-72].

The ATLAS collaboration [74] has recently estimated the accuracy of measuring the CP violating mixing angle $\phi_{\tau}$ defined via

$$
\mathcal{L}_{h \tau \tau}=g_{h \tau \tau}\left[\cos \left(\phi_{\tau}\right) \bar{\tau} \tau+\sin \left(\phi_{\tau}\right) \bar{\tau} i \gamma_{5} \tau\right] h
$$

With $3 \mathrm{ab}^{-1}$ at $\sqrt{s}=14 \mathrm{TeV}, \phi_{\tau}$ can be measured with a statistical precision between $\pm 18^{\circ}$ and $\pm 33^{\circ}$, depending on the energy resolution of the $\pi^{0}$ achieved in the reconstruction at high pile-up. 
Table X: Summary of the reach on $\lambda_{h h h}$ for various collider options. Given are the collider, the center-of-mass energy, the integrated luminosity and the precision that is expected to be achieved on $\lambda_{h h h}$. For all colliders, except FCC $\mathrm{F}_{e e}$, it is based on di-Higgs measurements. For $\mathrm{FCC}_{e e}$ it is based on the $\sqrt{s}$ dependence of higher order corrections to the $Z h$ cross section. In all cases, the luminosity quoted is that of the sum of the experiments. The values correspond to the luminosities given in Sec. II

\begin{tabular}{lcr}
\hline \hline Collider & $\sqrt{s}$ & $\frac{\Delta \lambda_{h h h}}{\lambda_{h h h}}(\%)$ \\
\hline \hline HL-LHC & $14 \mathrm{TeV}$ & \pm 50 \\
\hline ILC & $500 \mathrm{GeV}$ & \pm 17 \\
\hline CLIC & $3.0 \mathrm{TeV}$ & ${ }_{-7}^{+11}$ \\
\hline FCC $_{e e}$ & $240+365 \mathrm{GeV}$ & \pm 40 \\
\hline HE-LHC & $27 \mathrm{TeV}$ & $\pm(10-20)$ \\
\hline FCC $_{h h}$ & $100 \mathrm{TeV}$ & \pm 5 \\
\hline \hline
\end{tabular}

Table XI: A list of interesting theoretical questions, and a partial list of observables that are most relevant to making progress on these questions. $\kappa_{3}\left(\kappa_{\ell}\right)$ stands for third (first or second) generation couplings. $\mu_{4 f}$ stands for the processes $h \rightarrow S S \rightarrow \overline{f_{1}} f_{1} \overline{f_{2}} f_{2}$. For more details, see the text.

\begin{tabular}{|c|c|c|c|c|c|c|c|c|c|c|c|c|}
\hline Question & $\kappa_{V}$ & $\kappa_{3}$ & $\kappa_{g}$ & $\kappa_{\gamma}$ & $\lambda_{h h h}$ & $\sigma_{h Z}$ & $\mathrm{BR}_{\text {inv }}$ & $\mathrm{BR}_{\text {und }}$ & $\kappa_{\ell}$ & $\mu_{4 f}$ & $\mathrm{BR}_{\tau \mu}$ & $\Gamma_{h}$ \\
\hline Is $h$ Alone? & + & + & & & + & + & & & & + & & + \\
\hline Is $h$ elementary? & + & + & + & + & & + & & & & & & \\
\hline Why $m_{h}^{2} \ll m_{\mathrm{Pl}}^{2}$ ? & + & + & & & & & + & + & & + & & + \\
\hline 1st order EWPT? & & & + & + & + & + & & & & + & & \\
\hline CPV? & & $+(\mathrm{CP})$ & & & & & & & & & & \\
\hline Light singlets? & & & & & & & + & + & + & + & & + \\
\hline Flavor puzzles? & & + & & & & & & & + & & + & \\
\hline
\end{tabular}

\section{CONCLUSIONS}

A list of interesting theoretical questions, and a partial list of observables that are most relevant to making progress on these questions, are summarized in Table XI.

Our review of what can be learned from Higgs precision measurements led us to three important conclusions, independent of the particular experiments that will perform these measurements:

- The Higgs program, which is a guaranteed program of any future collider, touches some of the most significant open questions in particle physics and cosmology. In particular, this program may lead to progress in two puzzles of particle cosmology: dark matter and the baryon asymmetry.

- No Higgs related measurement will go unnoticed. Each of the production modes and each of the decay modes carries in it information that is relevant to important questions. Higgs decays to two bosons, two fermions, invisible modes, four fermions, as well as Higgs production by gluon-gluon fusion, $t \bar{t} h$, and di-Higgs production, all of these (and other measurements) are highly valuable for better understanding of particle physics and cosmology.

- For many of the interesting observables, the proposed future colliders significantly extend the sensitivity beyond that reachable by the HL-LHC alone.

\section{Acknowledgements}

We thank the members of the Scientific Policy Committee (SPC) of CERN for initiating discussions that led to this work, and for comments on the manuscript. We thank the participants of the workshop "Voyages Beyond the Standard Model II," and Gilad Perez and Georg Weiglein for useful discussions and comments on the manuscript. 
BH acknowledges support by the Deutsche Forschungsgemeinschaft (DFG, German Research Foundation) under Germany's Excellence Strategy - EXC 2121 "Quantum Universe" - 390833306. YN is the Amos de-Shalit chair of theoretical physics, and is supported by grants from the Israel Science Foundation (grant number 394/16), the United States-Israel Binational Science Foundation (BSF), Jerusalem, Israel (grant number 2014230), and the I-CORE program of the Planning and Budgeting Committee and the Israel Science Foundation (grant number 1937/12).

[1] G. Aad et al. [ATLAS Collaboration], Phys. Lett. B 716, 1 (2012) [arXiv:1207.7214 [hep-ex]].

[2] S. Chatrchyan et al. [CMS Collaboration], Phys. Lett. B 716, 30 (2012) [arXiv:1207.7235 [hep-ex]].

[3] F. Englert and R. Brout, Phys. Rev. Lett. 13, 321 (1964).

[4] P. W. Higgs, Phys. Rev. Lett. 13, 508 (1964).

[5] F. Bordry, M. Benedikt, O. Brüning, J. Jowett, L. Rossi, D. Schulte, S. Stapnes and F. Zimmermann, arXiv:1810.13022 [physics.acc-ph].

[6] P. Bambade et al., arXiv:1903.01629 [hep-ex].

[7] CEPC Study Group, arXiv:1811.10545 [hep-ex].

[8] M. Mangano et al., CERN-ACC-2018-0056.

[9] P. Huang, A. J. Long and L. T. Wang, Phys. Rev. D 94, no. 7, 075008 (2016) [arXiv:1608.06619 [hep-ph]].

[10] N. Craig, C. Englert and M. McCullough, Phys. Rev. Lett. 111, no. 12, 121803 (2013) [arXiv:1305.5251 [hep-ph]].

[11] D. Curtin, P. Meade and C. T. Yu, JHEP 1411, 127 (2014) [arXiv:1409.0005 [hep-ph]].

[12] J. Fan, M. Reece and L. T. Wang, JHEP 1508, 152 (2015) [arXiv:1412.3107 [hep-ph]].

[13] C. Frugiuele, E. Fuchs, G. Perez and M. Schlaffer, JHEP 1810, 151 (2018) [arXiv:1807.10842 [hep-ph]].

[14] A. M. Sirunyan et al. [CMS Collaboration], [arXiv:1809.05937 [hep-ex]].

[15] M. Aaboud et al. [ATLAS Collaboration], [arXiv:1809.06682 [hep-ex]].

[16] A. Robson and P. Roloff, arXiv:1812.01644 [hep-ex].

[17] T. Barklow, K. Fujii, S. Jung, R. Karl, J. List, T. Ogawa, M. E. Peskin and J. Tian, Phys. Rev. D 97, no. 5, 053003 (2018) [arXiv:1708.08912 [hep-ph]].

[18] P. Bechtle, S. Heinemeyer, O. Stål, T. Stefaniak and G. Weiglein, JHEP 1411, 039 (2014) [arXiv:1403.1582 [hep-ph]].

[19] T. Flacke, C. Frugiuele, E. Fuchs, R. S. Gupta and G. Perez, JHEP 1706, 050 (2017) [arXiv:1610.02025 [hep-ph]].

[20] ATLAS and CMS Collaborations, arXiv:1902.10229 [hep-ex].

[21] CMS Collaboration, Phys. Lett. B 785, 462 (2018) [arXiv:1805.10191 [hep-ex]].

[22] CMS Collaboration, JHEP 1710, 076 (2017) [arXiv:1701.02032 [hep-ex]].

[23] M. Aaboud et al. [ATLAS Collaboration], Phys. Lett. B 790, 1 (2019) [arXiv:1807.00539 [hep-ex]].

[24] CMS Collaboration, JHEP 1811, 018 (2018) [arXiv:1805.04865 [hep-ex]].

[25] ATLAS Collaboration, Phys. Lett. B 782, 750 (2018) [arXiv:1803.11145 [hep-ex]].

[26] CMS Collaboration, arXiv:1901.00174 [hep-ex].

[27] M. Cepeda et al. [Physics of the HL-LHC Working Group], arXiv:1902.00134 [hep-ph].

[28] ATLAS and CMS Collaborations, JHEP 1608, 045 (2016) [arXiv:1606.02266 [hep-ex]].

[29] A. Katz and M. Perelstein, JHEP 1407, 108 (2014) [arXiv:1401.1827 [hep-ph]].

[30] Y. Nir, Phys. Scripta T 158, 014005 (2013).

[31] A. Dery, A. Efrati, Y. Hochberg and Y. Nir, JHEP 1305, 039 (2013) [arXiv:1302.3229 [hep-ph]].

[32] K. Agashe et al. [Top Quark Working Group], arXiv:1311.2028 [hep-ph].

[33] A. Dery, A. Efrati, G. Hiller, Y. Hochberg and Y. Nir, JHEP 1308, 006 (2013) [arXiv:1304.6727 [hep-ph]].

[34] A. Dery, A. Efrati, Y. Nir, Y. Soreq and V. Susič, Phys. Rev. D 90, 115022 (2014) [arXiv:1408.1371 [hep-ph]].

[35] CMS Collaboration, arXiv:1809.10733 [hep-ex].

[36] ATLAS Collaboration, ATLAS note ATLAS-CONF-2018-031.

[37] T. K. Charles et al. [CLICdp and CLIC Collaborations], arXiv:1812.06018 [physics.acc-ph].

[38] J. Duarte-Campderros, G. Perez, M. Schlaffer and A. Soffer, arXiv:1811.09636 [hep-ph].

[39] Uta Klein, FCC-eh as a Higgs Facility. Talk at FCC Week, Amsterdam, 2018.

[40] ATLAS Collaboration, Phys. Rev. Lett. 120, 211802 (2018) [arXiv:1802.04329 [hep-ex]].

[41] G. Perez, Y. Soreq, E. Stamou, and K. Tobioka, Phys. Rev. D 92 no. 3, 033016 (2015), [arXiv:1503.00290]

[42] CMS Collaboration, Phys. Lett. B 744, 184 (2015) [arXiv:1410.6679 [hep-ex]].

[43] M. Aaboud et al. [ATLAS Collaboration], [arXiv:1812.11568 [hep-ex]].

[44] P. Azzi et al. [HL-LHC Collaboration and HE-LHC Working Group], arXiv:1902.04070 [hep-ph].

[45] ATLAS Collaboration, Eur. Phys. J. C 77, no. 2, 70 (2017) [arXiv:1604.07730 [hep-ex]].

[46] A. M. Sirunyan et al. [CMS Collaboration], JHEP 1806, 001 (2018) [arXiv:1712.07173 [hep-ex]].

[47] CMS Collaboration, Phys. Lett. B 763, 472 (2016) [arXiv:1607.03561 [hep-ex]].

[48] A. Efrati and Y. Nir, arXiv:1401.0935 [hep-ph].

[49] N. Arkani-Hamed, A. G. Cohen, E. Katz and A. E. Nelson, JHEP 0207, 034 (2002) [hep-ph/0206021].

[50] R. Contino, Y. Nomura and A. Pomarol, Nucl. Phys. B 671, 148 (2003) [hep-ph/0306259].

[51] K. Agashe, R. Contino and A. Pomarol, Nucl. Phys. B 719, 165 (2005) [hep-ph/0412089].

[52] G. F. Giudice, C. Grojean, A. Pomarol and R. Rattazzi, JHEP 0706, 045 (2007) [hep-ph/0703164]. 
[53] A. Thamm, R. Torre and A. Wulzer, JHEP 1507, 100 (2015) [arXiv:1502.01701 [hep-ph]].

[54] R. S. Gupta, H. Rzehak and J. D. Wells, Phys. Rev. D 86, 095001 (2012) [arXiv:1206.3560 [hep-ph]].

[55] D. Liu, I. Low and Z. Yin, arXiv:1809.09126 [hep-ph].

[56] P. W. Graham, D. E. Kaplan and S. Rajendran, Phys. Rev. Lett. 115, no. 22, 221801 (2015) [arXiv:1504.07551 [hep-ph]].

[57] D. J. H. Chung, A. J. Long and L. T. Wang, Phys. Rev. D 87, no. 2, 023509 (2013) [arXiv:1209.1819 [hep-ph]].

[58] C. Englert and M. McCullough, JHEP 1307, 168 (2013) [arXiv:1303.1526 [hep-ph]].

[59] S. Profumo, M. J. Ramsey-Musolf, C. L. Wainwright and P. Winslow, Phys. Rev. D 91, no. 3, 035018 (2015) [arXiv:1407.5342 [hep-ph]].

[60] A. V. Kotwal, M. J. Ramsey-Musolf, J. M. No and P. Winslow, Phys. Rev. D 94, no. 3 , 035022 (2016) doi:10.1103/PhysRevD.94.035022 [arXiv:1605.06123 [hep-ph]].

[61] J. Shu and Y. Zhang, Phys. Rev. Lett. 111, no. 9, 091801 (2013) [arXiv:1304.0773 [hep-ph]].

[62] A. Kobakhidze, L. Wu and J. Yue, JHEP 1604, 011 (2016) [arXiv:1512.08922 [hep-ph]].

[63] C. W. Chiang, K. Fuyuto and E. Senaha, Phys. Lett. B 762, 315 (2016) [arXiv:1607.07316 [hep-ph]].

[64] H. K. Guo, Y. Y. Li, T. Liu, M. Ramsey-Musolf and J. Shu, Phys. Rev. D 96, no. 11, 115034 (2017) [arXiv:1609.09849 [hep-ph]].

[65] R. Harnik, A. Martin, T. Okui, R. Primulando and F. Yu, Phys. Rev. D 88, no. 7, 076009 (2013) [arXiv:1308.1094 [hep-ph]].

[66] F. Demartin, F. Maltoni, K. Mawatari, B. Page and M. Zaro, Eur. Phys. J. C 74, no. 9, 3065 (2014) [arXiv:1407.5089 [hep-ph]].

[67] M. R. Buckley and D. Goncalves, Phys. Rev. Lett. 116, no. 9, 091801 (2016) [arXiv:1507.07926 [hep-ph]].

[68] S. Amor Dos Santos et al., Phys. Rev. D 96, 013004 (2017) [arXiv:1704.03565 [hep-ph]].

[69] F. P. Huang, P. H. Gu, P. F. Yin, Z. H. Yu and X. Zhang, Phys. Rev. D 93, no. 10, 103515 (2016) [arXiv:1511.03969 [hep-ph]].

[70] A. Hayreter, X. G. He and G. Valencia, Phys. Lett. B 760, 175 (2016) [arXiv:1603.06326 [hep-ph]]; Phys. Rev. D 94, 075002 (2016) [arXiv:1606.00951 [hep-ph]].

[71] D. Goncalves, K. Kong and J. H. Kim, JHEP 1806, 079 (2018) [arXiv:1804.05874 [hep-ph]].

[72] F. U. Bernlochner, C. Englert, C. Hays, K. Lohwasser, H. Mildner, A. Pilkington, D. D. Price and M. Spannowsky, Phys. Lett. B 790, 372 (2019) [arXiv:1808.06577 [hep-ph]].

[73] J. de Blas, R. Franceschini, F. Riva, P. Roloff, U. Schnoor, M. Spannowsky, J. D. Wells, A. Wulzer and J. Zupan CERN Yellow Reports: Monographs, Vol. 3/2018, CERN-2018-009-M (CERN, Geneva, 2018). [arXiv:1812.02093]

[74] The ATLAS collaboration, ATLAS-PHYS-PUB-2019-008. 